platform stabilized to the accuracy considered then it should be possible to observe the azimuth more accurately and more easily than the rate of change of altitude. The position is then determined by the solution of a spherical triangle in which two sides (altitude and declination) and the angle opposite one (the azimuth) are known; there are many practical methods of doing this and, in fact, a mechanical or electronic solution must be regarded as simple compared to the difficulty of stabilization and automatic observation.

\title{
The Accuracy of Astronomical Observations at Sea
}

\author{
from Commander J. M. Sharpey-Schafer, R.N.
}

THE statistical analysis in the Institute Working Party's report on the accuracy of astronomical observations at sea (this Journal, 10, 223 ) is a fine piece of work for which we should all be most grateful to Mr. Sadler and the staff of H.M. Nautical Almanac Office. Indeed it is perhaps not out of place to recall the traditions by which the Royal Observatory supported by the Government of the day has always helped navigation, and to express gratitude to the Astronomer Royal for permitting and encouraging the work.

One or two quite, interesting questions arise. There is a clear pattern in the observations of 50 per cent taken to $0: 2,25$ per cent to $0: 5$ and 25 per cent to whole I'. The table 'Accuracy of Sextant reading', 'Observers' Estimates', Table VIII and, more important, inspection of the forms, refer. Several errors given as 'Nil' on the forms seemed to indicate that within a whole I' was good enough. If all the observations had been taken to a whole $I^{\prime}$ or all had been taken to $0: 2$, it does not seem beyond the bounds of possibility that different answers would have been achieved. In the analysis they have not been split up. In that particular respect the analysis does not show that you might do better if you tried harder, although later it does analyse several observers who took a large number of sights and selects the better observers.

The next point is the number of shots. The report states that 'as far as the present observations are concerned the errors of a single shot appear to be just as accurate as those of the mean of 2 or 3'. Mr. Sadler has explained to me that this statement drawn from Table I, is given to explain why they are combined and subsequent precedures. Furthermore the report says 'practically all the observations based on the mean of 2 or 3 shots were made by only 2 observers'. Section 6 also refers. It seems important to emphasize that it is not a major conclusion of the analysis, indeed it appears to clash rather considerably with the methods of observation in surveying, astronomy and many other things; so much so that it just raises a slight doubt as to whether something has crept into the whole trial which might be swamping the results. However, what is clear is that the Working Party in its future work ought to test the subject of the number of shots from $1-7$ against high and low-power magnification in a different way, which might be done simply by a few observers on shore in a day.

Similarly a minor point is the State of the Sea not showing any significance, 
but apparently only a few small ships sent in observations and only few of those in poor sea conditions.

Over the observer's estimate of the accuracy of position by shore fix, \&c., to I $: 0$ or better there was usually no evidence to assess them on, but an inspection of some of the forms revealed an occasional remark such as 'Bearings at 30 miles.' Taking into account that gyros are only designed to an accuracy of $2^{\circ}$, magnetic compasses much the same, the difficulty of being quite sure of the objects at a distance over 10 miles, a fair proportion of narrow cuts and running fixes and some lack of precision, small we hope, in the geographical coordinates of certain coasts, it is not just too happy to accept the mariner's own estimate of the accuracy of his position outside ro miles. Inside 10 miles, it seems to be generally agreed that abnormal refraction may play a part. This was remarked on in the Journal, 6, 367 .

The next point is the errors of Reduction or Calculation. It seems that 42 out of 97 observations sampled agreed numerically to within $0: 5$ or less on recalculation. And frankly within the limitations of the tables as at present constituted, $0: 5$ ought to be a better criterion than $I: 0$. Some explanations are noted, and the I 2 apparently uncorrected for dip and refraction seem acceptable, but it is not clear whether they are included or not in the 50 out of 97 that are consistent to within ${ }^{\prime}$ '. Perhaps $\mathrm{Mr}$. Sadler will tell us. But in any case the calculation errors are worrying. The errors of calculation have not been sorted out by the analysis or removed from the 1858 observations. Firstly one very seriously wonders, indeed, whether this calculation error might not by its size be rather hiding the effects of most of the other causes of error. It will not be realized by the great audience of navigators that in other fields where statistical analysis is applied, and where hundreds of fairly similar trials are compared in summarizing scientific papers, the differences between trials are remarkable, to the extent that it is lucky to get a quarter of the items confirmed. Secondly apart from this analysis something seems rather dreadfully wrong about calculations for sights. Ponderous no doubt. 30 or 40 per cent of sights calculated wrong, ought to make us sit up with a bang. I may add that I always held that it was so for my own and everybody else's sights, and for a specialist accuracy the only thing was to check the lot right through. It would seem to be rather a major conclusion, to be emphasized, that calculation errors will need considerable investigation by other means. It seems fairly well known in the background, that probably I' would suit most navigation by sights most of the time, and what a saving there could be in tables and calculations. This is supported in these observations by the vast majority who take one shot, the $5^{\circ}$ per cent bias to a whole $I^{\prime}$ and $\frac{1}{2}^{\prime}$, and the bias to a sextant magnification of $2 \cdot 5-3 \cdot 0$, i.e. a star telescope, presumably for suns as well. For specialist needs when attempting to get inside $I^{\prime}$ other tables are available.

It may be noted that the analysis does not show what really contributes to the large errors, except 'Horizon'. It is known in astronomy and surveying sights on shore, that by eliminating the sea horizon much greater accuracy is comparatively easy. That is if we ever want such accuracy. But the sea horizon imposes several other limitations, at night and in poor conditions. Although the accelerations of a ship's movement are far more violent than an aircraft and have always been held to make a bubble sextant fairly impractical, it is suggested that the Working Party should review the subject of horizon.

$\mathrm{Mr}$. Parker tells us that if the 50 per cent error of a single position line is $0: 7$, then in the best case of three position lines cutting in a cocked hat of an equi- 
lateral triangle, the $5^{\circ}$ per cent error of the fix would be $1^{\prime} \circ$ (see Journal, 5 , 24I). This seems reasonable, but it is interesting to note that the probable error of such a fix is larger than for the position lines.

It would be wrong to think that statistical analysis is all nonsense, far from it, but like any other diagnostic method it has its difficulties and pitfalls. Refinement of the observations for one, and in that connection it is understood that among statisticians a need is felt to control the observations rather closely. Another, in this case, is that it is often difficult to say clearly what is really contributing to the errors. This analysis is excellent in showing up some of these difficulties and providing some interesting though rough results. Rome was not built in a day. It is to be hoped that the Working Party will continue and investigate by other and separate trials certain aspects which are still in doubt.

To end on a brighter note is the story of three statisticians who got drunk three days running, and the only thing they could correlate as common between them was the soda water, which must have made them drunk.

The substance of Commander Sharpey-Schafer's note was raised in the discussion on 21 June (when the Working Party's Report was presented to an Ordinary Meeting). Mr. Sadler then commented:

Commander Sharpey-Schafer quoted that something like 25 per cent of observations seemed to be taken only to the nearest minute. I am not quite sure where he got his figures from but the actual figures are as follows: 76 observers worked to $0 ! 2,44$ worked to $0: 5,3$ I gave no information. I think he has assumed that those who gave no figures worked to the nearest minute only; he may be right, but the point I wish to make is that, when we are talking in terms of errors of the present size, very little difference is caused by rounding off to the nearest minute. In fact the errors themselves are often grouped in this way for ease of analysis.

Secondly, I would like to support Commander Sharpey-Schafer in his remarks regarding the extreme importance of the implication of a large proportion of errors in the reductions. It is most disturbing, but it is difficult to know what to do; the Working Party would have liked to have taken all 3000 observations and reduced them completely afresh. This could be done, but it would be very laborious ; moreover, although the actual calculation is not difficult, the interpretation of the difference between the two calculations is extremely difficult, even when you yourself have made no mistake. You may spend one or two hours trying to find the reason for each discrepancy, and there is no certainty that the analysis will indicate any particular causes that can be eradicated.

The only other point I would like to comment on is how this, and similar, investigations can lead to an improvement in navigational practice. The first object, which I think has been obtained, is to find out what is being done now. There are two ways in which this practice can then be improved: firstly to simplify procedures, without decreasing the accuracy which appears satisfactory for most purposes, and thus to reduce the chance of blunders; and secondly, where the greatest possible accuracy is required, to separate out the causes of the errors in order to eliminate or to reduce them. There is still plenty of work for the Working Party. 